\title{
Potassium-Doped Para-Terphenyl: Structure, Electrical Transport Properties and Possible Signatures of a Superconducting Transition
}

\author{
Nicola Pinto ${ }^{1,2}$, Corrado Di Nicola ${ }^{3}$, Angela Trapananti ${ }^{1}$, Marco Minicucci ${ }^{1}$, Andrea Di Cicco ${ }^{1}$, \\ Augusto Marcelli ${ }^{4}$, Antonio Bianconi ${ }^{5,6}$, Fabio Marchetti ${ }^{3}$ and Claudio Pettinari ${ }^{7}$ \\ and Andrea Perali $8, *$ \\ 1 Physics Division, School of Science and Technology, University of Camerino, 62032 Camerino, Italy; \\ nicola.pinto@unicam.it (N.P.); angela.trapananti@unicam.it (A.T.); marco.minicucci@unicam.it (M.M.); \\ andrea.dicicco@unicam.it (A.D.C.) \\ 2 Advanced Materials Metrology and Life Science Division, INRiM (Istituto Nazionale di Ricerca Metrologica), \\ 10135 Turin, Italy \\ 3 Chemistry Division, School of Science and Technology, University of Camerino, 62032 Camerino, Italy; \\ corrado.dinicola@unicam.it (C.D.N.); fabio.marchetti@unicam.it (F.M.) \\ 4 INFN_Laboratori Nazionali di Fisica Nucleare, 00044 Frascati, Italy; augusto.marcelli@lnf.infn.it \\ 5 RICMASS, Rome International Centre for Material Science Superstripes, 00185 Rome, Italy; \\ antonio.bianconi@ricmass.eu \\ 6 Institute of Crystallography, IC, CNR, Monterotondo, 00015 Rome, Italy \\ 7 Chemistry Unit, School of Pharmacy, University of Camerino, 62032 Camerino, Italy; \\ claudio.pettinari@unicam.it \\ 8 Physics Unit, School of Pharmacy, University of Camerino, 62032 Camerino, Italy \\ * Correspondence: andrea.perali@unicam.it
}

Received: 30 September 2020; Accepted: 20 November 2020; Published: 1 December 2020

\begin{abstract}
Preliminary evidence for the occurrence of high- $T_{C}$ superconductivity in alkali-doped organic materials, such as potassium-doped p-terphenyl (KPT), were recently obtained by magnetic susceptibility measurements and by the opening of a large superconducting gap as measured by ARPES and STM techniques. In this work, KPT samples have been synthesized by a chemical method and characterized by low-temperature Raman scattering and resistivity measurements. Here, we report the occurrence of a resistivity drop of more than 4 orders of magnitude at low temperatures in KPT samples in the form of compressed powder. This fact was interpreted as a possible sign of a broad superconducting transition taking place below $90 \mathrm{~K}$ in granular KPT. The granular nature of the KPT system appears to be also related to the $20 \mathrm{~K}$ broadening of the resistivity drop around the critical temperature.
\end{abstract}

Keywords: doped organic molecule; Raman; electronic transport properties; superconductivity

\section{Introduction}

Molecules with extensive systems of $\pi$-bonds are the main components of the so-called molecular superconductors [1]. Potentially interesting are aromatic hydrocarbon molecules, consisting of connected benzene rings, since doping by alkali metals of their solid phase provides the superconductivity [1]. The first discovery of such superconductivity was made for K-doped picene [2]. Aiming to find aromatic compounds promising for high $T_{C}$ superconductivity, attention was focused on $p$-terphenyl. The structure of the undoped crystalline $p$-terphenyl $\mathrm{C}_{18} \mathrm{H}_{14}$ ( $p$-terphenyl in the following) has attracted long standing interest [3-10]. This molecule is made of three phenyl rings 
connected by single C-C bond in para position. The room temperature (RT) structure was described as a packing of nanoribbons, made of $p$-terphenyl molecules, in the crystallographic $b$ direction. Therefore, the structure of the molecule can be interpreted as a realization of a nanoscale architecture of low dimensional systems [11,12] described as a self-organized array of polymeric quantum stripes [13,14]. While pristine $p$-terphenyl is electrically insulating, it behaves as a metal upon K-doping [15]. Recent experimental signatures of superconductivity in alkali-doped para-oligophenyl compounds [16-18] and in $p$-terphenyl $\mathrm{K}_{x} \mathrm{C}_{18} \mathrm{H}_{14}$ [19-22] in particular, has attracted considerable interest. Increasing the $\mathrm{K}$ concentration toward $\mathrm{K}_{3} \mathrm{C}_{18} \mathrm{H}_{14}$ and lowering the temperature, the K-doped $p$-terphenyl (KPT) shows signatures of superconductivity in the magnetic response, consisting of a weak Meissner shielding effect [21]. The reported range of superconducting critical temperature, $T_{C}$, goes from a few $\mathrm{K}$ [15] to $123 \mathrm{~K}$ [21], where the repeatability of $T_{C}$ is difficult since this remains a complex quantum material characterized by inhomogeneous concentrantion of dopants and a competition of multiple coexisting phases. The dc and ac magnetic susceptibility measurements reported by Wang et al. demonstrate that KPT is a type-II superconductor [15]. In the KPT compound studied in Ref. [15] with a $T_{C}=7.2 \mathrm{~K}$, characterized by a moderate level of K-doping, the critical magnetic fields extrapolated at zero temperature are $H_{c 1}(0)=163$ Oe and $H_{c 2}(0)=1317 \mathrm{Oe}$, from which it results a magnetic penetration depth $\lambda=76 \mathrm{~nm}$ and a coherence length $\xi=50 \mathrm{~nm}$, by applying Ginzburg-Landau relations. In the KPT compound showing a much higher $T_{C}$ of the order of $120 \mathrm{~K}$, with an higher level of $\mathrm{K}$-doping, there is evidence for an upper critical magnetic field higher than $3 \mathrm{~T}$, as expected for high- $T_{C}$ type-II superconductors [21]. An independent detection of superconductivity at $107 \mathrm{~K}$ in KPT compounds by magnetic measurements was reported in Ref. [23]. On the other hand, in Ref. [24] no signs of a superconducting transition have been detected by magnetic susceptibility and resistivity measurements in a KPT compound. Indeed, a metallic behaviour was found below $150 \mathrm{~K}$. However, high resolution angle resolved photoelectron spectroscopy (ARPES) technique measurements, realized on potassium surface-doped $p$-terphenyl demonstrate the opening of a sizeable gap in the excitation spectrum, of the order of $\Delta \sim 12 \mathrm{meV}$, persisting up to $120 \mathrm{~K}$ [25]. The gap is almost independent on temperature in the range $10-60 \mathrm{~K}$. Above $T=60 \mathrm{~K}$ the gap fills in: the scattering rate becomes of the same order of the energy gap. At $T=120 \mathrm{~K}$ the photoemission spectrum resembles the one of a normal metal. The existence of a gapped spectrum, with an energy gap of $11 \mathrm{meV}$ close to that detected by ARPES, was confirmed by scanning tunneling microscopy (STM) applied to monolayer KPT [26]. The gap closes around $T=50 \mathrm{~K}$, even though no response to a magnetic field up to $11 \mathrm{~T}$ was observed. ARPES and STM experiments point toward a superconducting gap and coherent Cooper pairing below $T=60 \mathrm{~K}$, while in the range 60-120 K Cooper pairing could be local and incoherent: the pseudogap physics accompanied with strong fluctuations could emerge in a similar way to underdoped cuprate superconductors and ultracold fermions in the crossover regime of the BCS-BEC crossover [27-30]. Multiband effects combined with the BCS-BEC crossover could also contribute in determining the optimal parameter configuration for high- $T_{C}$ superconductivity in this material [31,32]. A possible theoretical scenario of the high- $T_{C}$ in these systems is based on resonance effects in a superlattice of quantum stripes associated with the quasi-1D structure of the $p$-terphenyl with coexisting polarons and Fermi particles $[13,33]$. The vibrational properties and the geometrical features of the lattice should play a key role in this system in the formation of Cooper pairs induced by strong electron-phonon coupling with high energy phonons. The role of strong electronic correlation and of the multiband electronic structure in determing the superconducting properties of KPT has been discussed in Ref. [34].

In this article we report the experimental investigation of the conducting and possible superconducting behaviour of K-doped $p$-terphenyl samples, obtained by our independent synthesis. The structure of the material was studied by Raman spectroscopy as a function of the temperature and correlated with the electrical behaviour. The huge drop of the resistivity below $100 \mathrm{~K}$, occurring in about $22 \mathrm{~K}$, suggests a granular-like superconducting behaviour. 


\section{Experimental}

p-terphenyl and potassium metal (99\% purity) have been purchased from Sigma-Aldrich.

The $p$-terphenyl was purified by sublimation, to achieve a purity above $99.9 \%$. The potassium was purified by distillation. Using a glove box, potassium $3 \mathrm{~mol},(117 \mathrm{mg})$ was cut in little pieces and mixed with $p$-terphenyl $1 \mathrm{~mol},(230 \mathrm{mg})$. The mixture was sealed in a glass tube under argon atmosphere and heated at $503 \mathrm{~K}$ for $2 \mathrm{~h}$. An oxygen and water sensitive black powder was obtained.

The exposure to atmosphere of doped $p$-terphenyl gives rise to its decomposition into a whitish hygroscopic mixture of $p$-terphenyl and potassium hydroxide, mainly due to the presence of moisture in air. As well known in the literature [35], similar potassium-doped aromatic compounds react immediately with water affording an aqueous solution of $\mathrm{KOH}$ and the undoped aromatic compound with evolution of gaseous hydrogen. For this reason we have decided to take advantage of reactivity by analyzing the amount of potassium ion which is present in the final solution obtained by reaction of our doped sample with water, using atomic absorption spectroscopy (AAS), by a Perkin Elmer mod. $1100 \mathrm{~B}$, and then go back to the molar ratio between potassium and $p$-terphenyl in the starting doped material. $7.2 \mathrm{mg}(0.0239 \mathrm{mmol})$ of doped $p$-terphenyl was suspended in $50 \mathrm{~mL}$ of distilled water and left to react at room temperature under stirring for $20 \mathrm{~min}$, the suspension was then filtered off and analysed. The analysis has revealed $34.4 \mathrm{ppm}$ of present in the final solution, corresponding to $1.72 \mathrm{mg}$ of potassium in $7.2 \mathrm{mg}$ of doped $p$-terphenyl sample, so the ratio (potassium):( $p$-terphenyl) in milligrams is (1.72):(5.48) corresponding to a millimolar ratio (1.85):(1.00), in agreement with the presence of about 2 potassium cations for each $p$-terphenyl molecule, as shown in Figure 1.
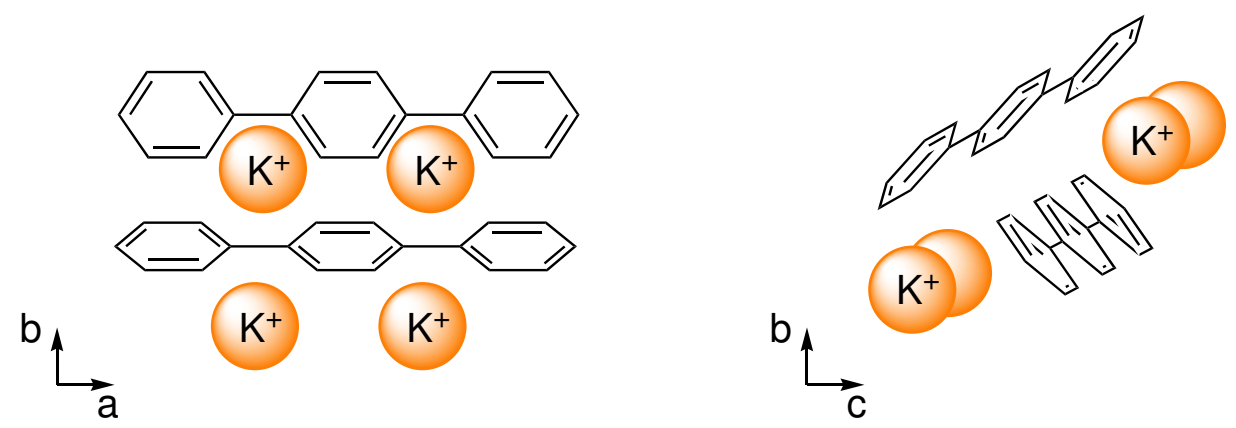

Figure 1. Example of doping with two $\mathrm{K}$ atoms of a $p$-terphenyl molecule, $\mathrm{K}_{2} \mathrm{C}_{18} \mathrm{H}_{14}$. The tilted geometry results from the first principle calculations of Ref. [36].

Raman spectra were measured using a micro-Raman setup including a Czerny-Turner spectrometer (iHR320 Horiba Scientific) equipped with 1800 grooves/mm grating and an open space microscope to accommodate the cell for low temperature measurements (Linkam THMS600). Experiments were performed with an excitation wavelength of $532 \mathrm{~nm}$. The laser power was set at less than $1 \mathrm{~mW}$ to avoid sample damage. To measure the KPT spectra, the synthesized powder was loaded into an especially designed sample container sealed under Ar atmosphere to prevent any exposure to air and moisture.

Electrical characterization was carried out on compressed KPT powder in the range 75-115 MPa. The powder was inserted into a teflon block, with a cylindrical hollow (with a diameter of $8 \mathrm{~mm}$ or $12.8 \mathrm{~mm}$ ), and compressed by two air-tight copper leads, part of a mechanical vise suitably designed for this purpose, resulting in a KPT flat disk inside the teflon block. All electrical measurements have been done keeping the KPT disk inside the mechanical vise. To each copper lead, working as electrode in tight contact with the KPT disk base, two couples of electrical cables implement the 4 -wires geometry of measure. The minimum resistance value achievable by our measuring system was checked to be $\simeq 9 \times 10^{-5} \Omega$, about 1.5 orders of magnitude lower than the lowest resistance detected in the KPT material. Sample cooling was achieved by two different equipments, using for each a specific mechanical vise (as that described above) keeping disks and electrodes under a tight electrical 
contact during the measurement. Mechanical vises turn out to be also necessary to keep good electrical contacts among powder grains. In one of the two equipments, the mechanical vice was located inside a small open air dewar and liquid nitrogen $\left(\mathrm{LN}_{2}\right)$ slowly poured. This system was used to measure the resistivity, $\rho(T)$, during the cooling from $300 \mathrm{~K}$ to about $80 \mathrm{~K}$, occurred in about $15 \mathrm{~min}$. This apparatus was preferred for resistivity measurement, not requiring a temperature stabilization, since it has allowed to keep as short as possible the time elapsed from the fabrication of KPT disks to the beginning of the electrical characterization, generally less than one hour. The sample temperature was measured by a thermocouple and monitored by an Eurotherm mod. 3216 temperature controller. In the second equipment, a He closed cycle cryostat has allowed to investigate the current-voltage characteristics at several fixed temperatures (for details see [37,38]). Measurements have been started upon cooling at $\simeq 3 \mathrm{~K}$. For K doped $p$-terphenyl disks, the resistivity and the current-voltage characteristics (I-V) have been carried out sourcing a dc current by a Keithley mod. 220 and measuring the voltage drop with a Keithley mod. 2000. Conversely, for undoped $p$-terphenyl disks, due to the high resistance of the material, both resistivity and I-V characteristics have been measured in the two contacts geometry, by using a pico-ammeter Keithley mod. 6487, applying a constant voltage and measuring the current.

\section{Results and Discussion}

\subsection{Raman Spectroscopy}

Raman spectra of undoped $p$-terphenyl have been measured in the temperature range 298-92 K and are shown in Figure 2. Fluorescence background is subtracted in all the spectra. All the peaks have been assigned to the molecular (high frequency) and lattice modes (low frequency, Raman shift $<200 \mathrm{~cm}^{-1}$ ) [39]. As shown in Figure 2 (inset), the band at $\sim 1280 \mathrm{~cm}^{-1}$, assigned to C-C inter-ring stretching, splits on lowering the temperature, as reported in previous studies [40,41]. A similar behaviour is observed for the band at $\sim 230 \mathrm{~cm}^{-1}$ associated with $\mathrm{C}-\mathrm{C}$ bending modes. Such changes are related to the disorder-order transition occurring in the $p$-terphenyl lattice at about $190 \mathrm{~K}$ from a monoclinic structure to a triclinic structure [3,4,42]. At room temperature, each $p$-terphenyl molecule has a conformation planar on average, with thermal vibrations of the phenyl rings due to competing steric repulsion between $\mathrm{H}$ atoms (favouring a ring tilt) and delocalized $\pi$ electrons (promoting the planar geometry). Decreasing the temperature, each molecule changes into a configuration in which the external phenyl rings are tilted and such molecular re-arrangement results in a different lattice symmetry.

p-terphenyl doping by potassium (electron donor), induces important differences in the Raman spectrum with respect to the pristine compound (Figure 3). The low frequency lattice modes disappear, possibly indicating reduced crystallinity of the doped sample. In the high frequency range, between $1100 \mathrm{~cm}^{-1}$ and $1600 \mathrm{~cm}^{-1}$, shifts of existing peaks and additional bands can be seen, related to strong modifications of the molecular geometry and to charged defects induced by doping. The two peaks at $1593 \mathrm{~cm}^{-1}$ and $1605 \mathrm{~cm}^{-1}$ in the $p$-terphenyl (intra-ring C-C stretching) tend to merge into one only, downshifted at $1588 \mathrm{~cm}^{-1}$. The peak at $1277 \mathrm{~cm}^{-1}$ in the pristine corresponds to the one at $1348 \mathrm{~cm}^{-1}$ in the doped compound. The upshift of such band assigned to inter-ring C-C stretching, indicates that the length of the $\mathrm{C}-\mathrm{C}$ bond decreases. Other observed bands originate from Raman-inactive modes of the neutral $p$-terphenyl. These effects are all related to the structural changes occurring upon doping: the molecular structure changes from benzenoid to quinoid and the phenyl rings are almost coplanar with nearly zero tilting between adjacent phenyl rings $[43,44]$. 


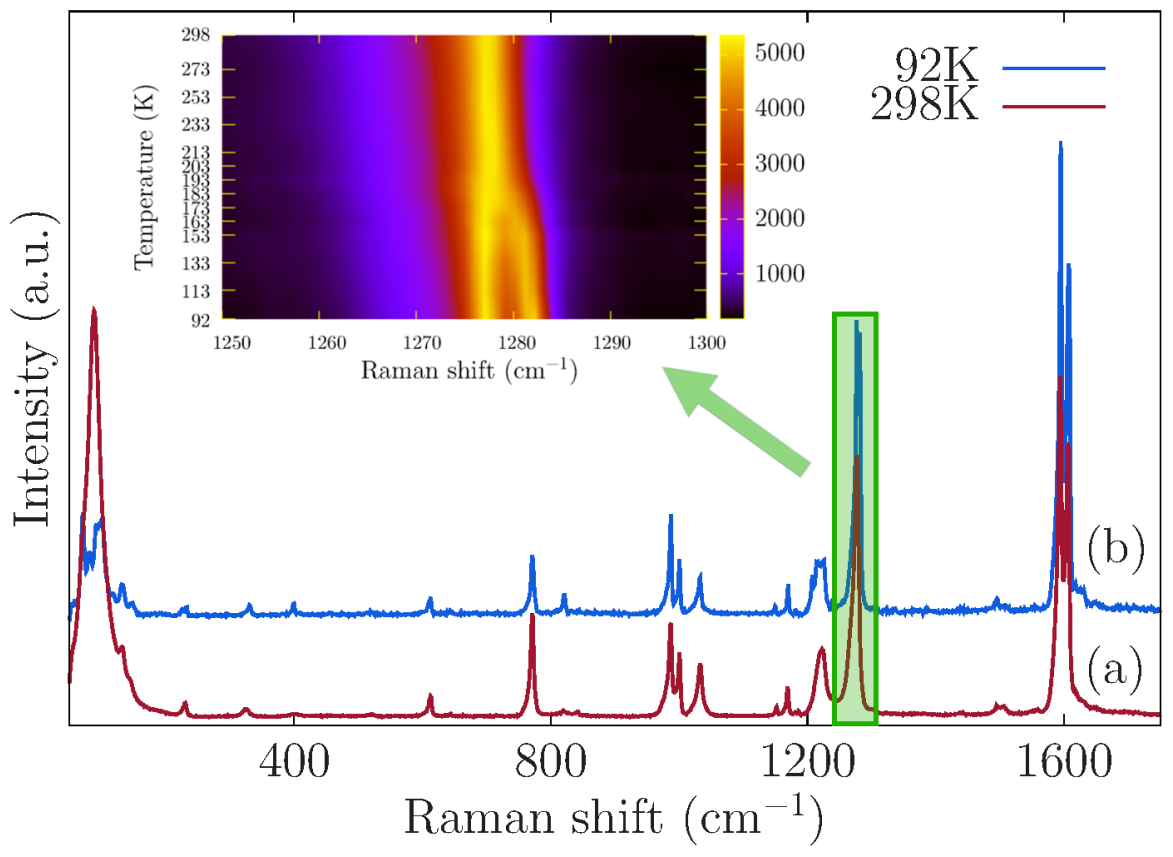

Figure 2. Raman spectra of pristine $p$-terphenyl at room temperature (a) and $92 \mathrm{~K}(\mathbf{b})$. Curves have been shifted for clarity. The inset shows the evolution of the Raman band at $\sim 1280 \mathrm{~cm}^{-1}$ (C-C inter-ring stretching) as lowering the temperature. The right scale (colour code) represents the intensity.

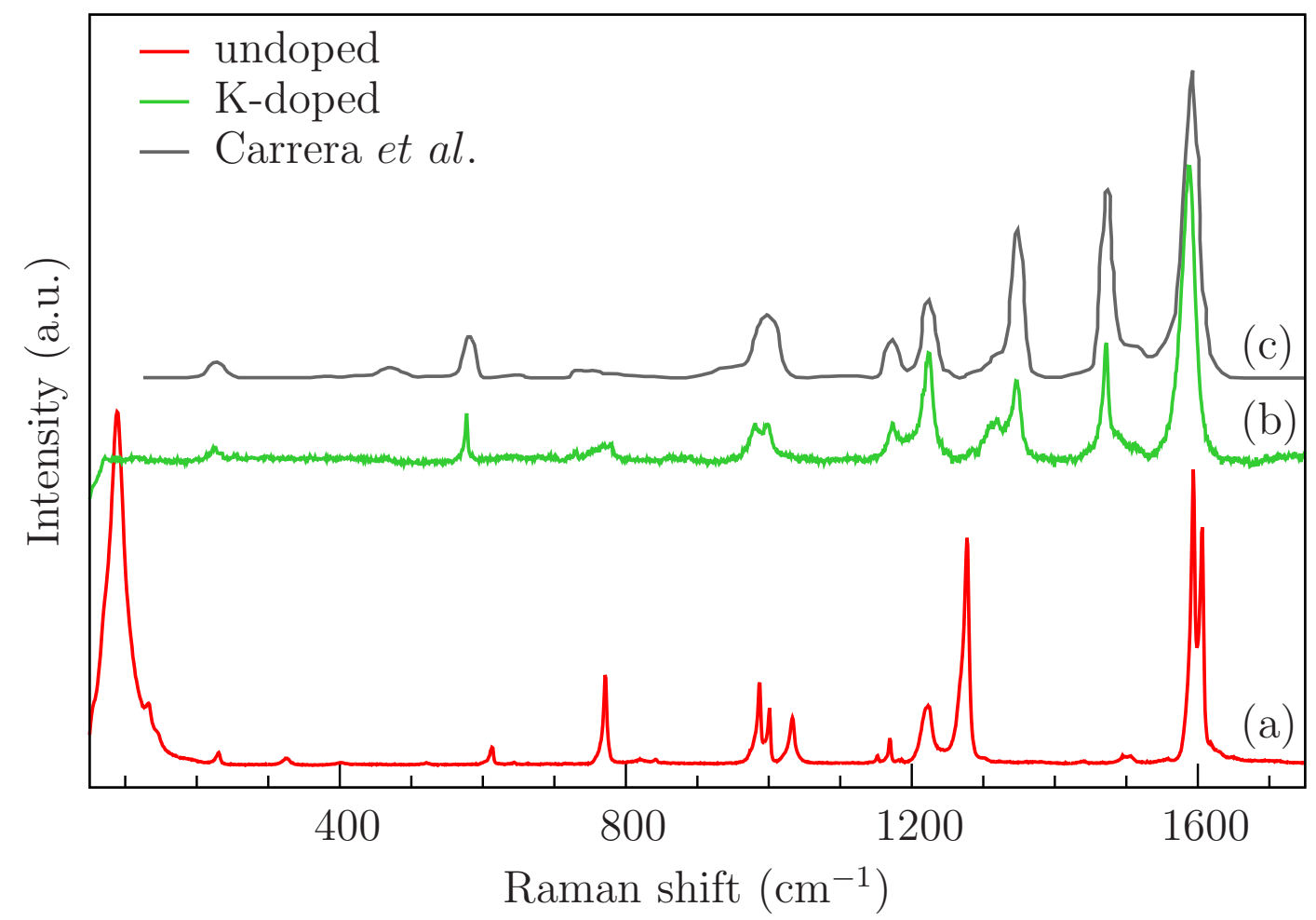

Figure 3. Raman spectra of undoped (a) and K-doped (b) p-terphenyl measured at RT. Curves have been shifted for clarity. For comparison the Raman spectrum for the K-doped para-terphenyl as digitized from Ref. [24] is also shown (c).

The main Raman bands are in very good agreement with those reported in Ref. [24,45] as well as with the one reported in Ref. [15], providing evidence of a successful doping of our KPT sample. 
However, Raman spectroscopy does not allow quantifying the level of K-doping of the $p$-terphenyl molecule, known to affect the superconducting properties of KPT [13]. In fact, for a KPT material synthesised with different $\mathrm{K}$ to $p$-terphenyl mole ratio, but showing very similar Raman spectra, the group of Ref. [13] has reported $T_{c}=7.2 \mathrm{~K}$ for a starting mole ratio of $2: 1 ; T_{c}=43 \mathrm{~K}$ and $T_{c}=123 \mathrm{~K}$ in Ref.s [18] and [19], respectively, for an higher mole ratio of 3:1.

In previous literature on doped conducting polyphenyls [45-47], Raman spectroscopy was extensively used to characterize structural changes and to selectively identify bands corresponding to polarons and bipolarons by changing the excitation wavelength from $514.5 \mathrm{~nm}$ to $1064 \mathrm{~nm}$ [44]. The formation of bipolarons in KPT compounds is considered to be the driving force inducing the structural transition from benzenoid to quinoid associated with the deformation of the internal benzene ring [15]. The Raman band at $1473 \mathrm{~cm}^{-1}$ in our KPT (see our Figures 3 and 4) was considered to be the fingerprint for the formation of bipolarons. Direct Bose-Einstein condensation of bipolarons at high densities was proposed as a possible mechanism for high- $T_{C}$ superconductivity in KPT compounds $[15,21]$. Original proposals of high- $T_{C}$ superconductivity driven by bipolarons have been reported in Refs. [48,49]. To correlate structural changes and formation of localized charged states, with the resistivity measurements at low temperature, Raman spectra of KPT have been measured also at different temperatures down to $108 \mathrm{~K}$. The spectra (Figure 4) do not evidence any change of the Raman activity, indicating that the doped material maintains the molecular structure upon cooling and new polaronic or bipolaronic bands are not detected at low temperatures. The inter-ring C-C stretching band, close to $1348 \mathrm{~cm}^{-1}$, does not split lowering the temperature, in contrast to what was observed for the undoped compound possibly indicating an already planar configuration for the doped sample retained for the whole temperature range. The dopants decrease the torsional mobility of the central ring as a result of the reduced length of the $\mathrm{C}-\mathrm{C}$ bonds between the rings. Just the spectrum at $T=203 \mathrm{~K}$ in Figure 4 shows weak lattice peaks and one peak at $\sim 1280 \mathrm{~cm}^{-1}$ (marked with a star) which have been attributed to a small fraction of undoped sample, intercepted by the microbeam $\left(\sim 2 \times 2 \mu \mathrm{m}^{2}\right)$ used for micro-Raman measurements. This indicates that the powder is not completely homogenous and there are grains of micrometric size of undoped $p$-terphenyl in our sample.

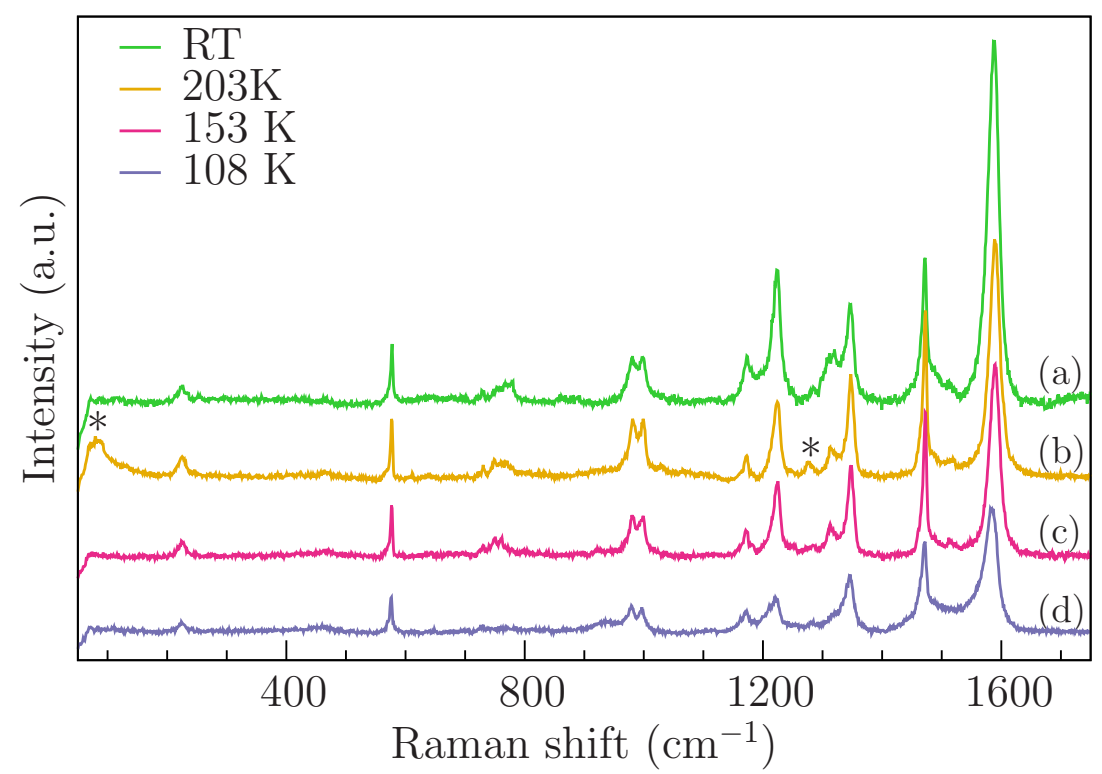

Figure 4. Raman spectra for K-doped $p$-terphenyl measured at different temperatures, from RT (a) down to $108 \mathrm{~K}$ (d). At $203 \mathrm{~K}$, two additional features, not visible in the other plotted spectra, have been marked by a star. Curves have been shifted for clarity. 


\subsection{Electrical Characterization}

The electrical properties of polycrystalline films of undoped $p$-terphenyl, a few micrometres thick, have been investigated so far by several authors [50-53]. Depending on the temperature range and/or the intensity of the applied electric field, several transport mechanisms have been reported such as, field emission, Poole-Frenkel, variable range hopping, etc. [50-53]. In a p-terphenyl film, both metallic and insulating behaviours have been detected, above and below $\sim 80 \mathrm{~K}$, respectively [52] while, in the range from $110 \mathrm{~K}$ to about RT, Lipinski et al. have established that hopping is the main conduction mechanism [50]. These features are consistent with a high density of traps or donor-acceptor-like sites in the band-gap of the material, causing a charge carrier transport characterized by jumps among localized states [50-52]. We have investigated the electrical properties of either undoped or K-doped $p$-terphenyl disks. In undoped $p$-terphenyl, resistivity values confirmed the insulating nature of the pristine material with a $\rho_{R T}>10^{16} \Omega \mathrm{cm}$ that, in the sensitivity limit of the whole measuring apparatus (instruments, cryogenic cables, etc.), remained practically constant also at lower $T$.

Upon doping, KPT samples with a RT resistivity greater than $\sim 10^{4} \Omega \mathrm{cm}$ do not show a metallic behaviour at low temperature indicating an unsuccessful doping process. On the contrary, when the RT resistivity is below $\sim 10^{2} \Omega \mathrm{cm}$ a completely different $\rho_{R T}(T)$ behaviour is detected at low temperature.

In detail, for the class of KPT samples with low $\rho_{R T}$, lowering $T$ from RT, resistivity rises something more than one order of magnitude, saturating to $\simeq 80 \Omega \mathrm{cm}$ at $130-150 \mathrm{~K}$. Spurious irregularities, as peaks and bumps, are visible along this branch of the curve, originated by a rapid change of the pressure exerted by the mechanical vice on the KPT disk. The observed features in $\rho(T)$ are related to the grainy nature of KPT powder used to fabricate disks. Below $\approx 130 \mathrm{~K}, \rho(T)$ becomes metallic-like indicating an enhanced contribution to the conduction by electrons. The initial slow decrease of $\rho(T)$ is followed by a steeper fall at $\approx 100 \mathrm{~K}$ that, in addition to the residual value of less than $10^{-2} \Omega \mathrm{cm}$, below $30 \mathrm{~K}$ (Figure 5), recalls that of a superconducting transition. In a range of $\simeq 22 \mathrm{~K}$, a drop of resistivity by $\approx 4$ orders of magnitude was observed in our measurement. This resistivity drop shares some features of granular superconductivity, as discussed later.

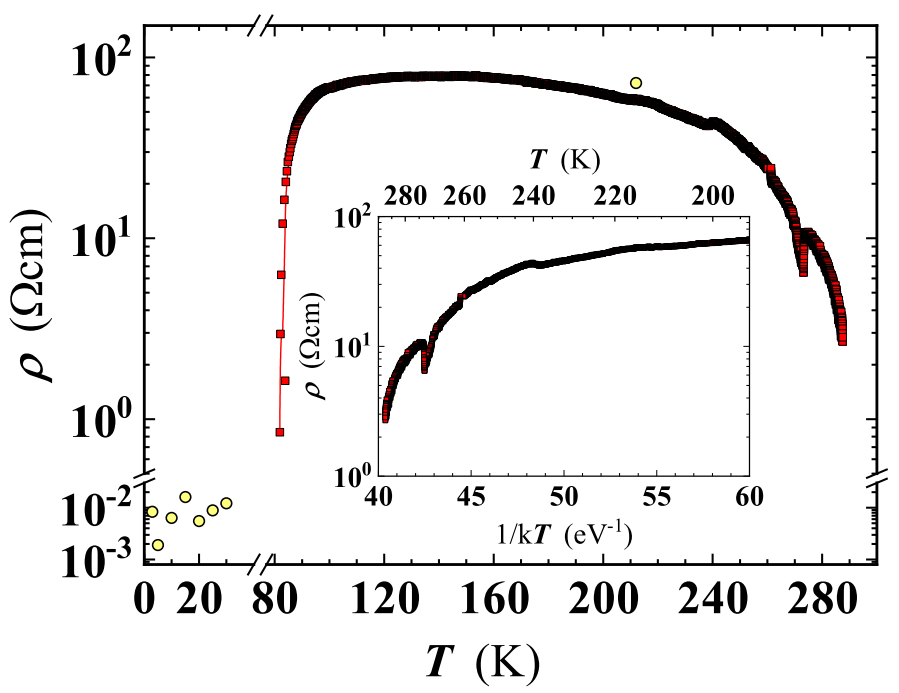

Figure 5. Resistivity as a function of temperature for a doped KPT disk. Squares: $\rho(T)$ values measured carrying out the measurement at constant current of $1 \mu \mathrm{A}$. Circles: $\rho(T)$ values derived from I-V characteristics (see Figure 6) under similar conditions of measure. Inset: Arrhenius plot of the high temperature branch of the resistivity curve. 


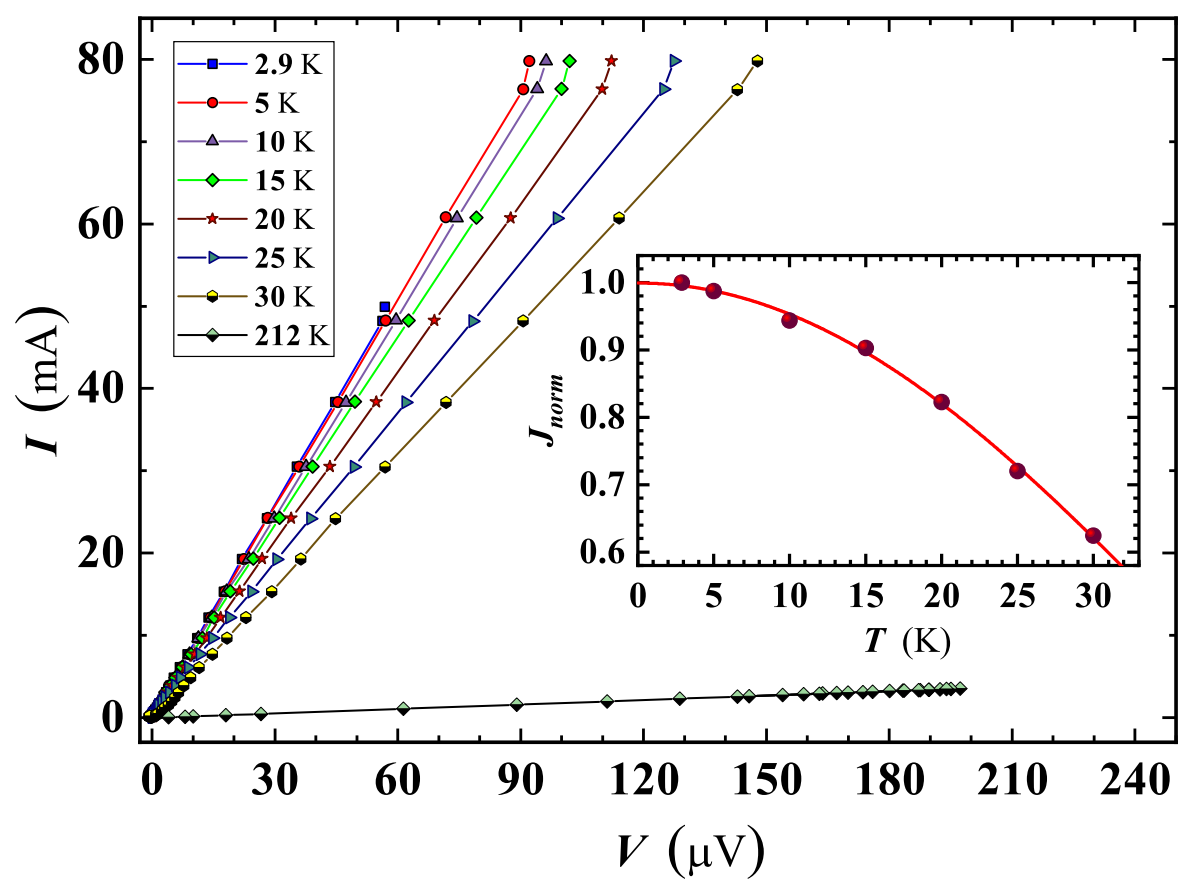

Figure 6. Current-voltage characteristics of a doped KPT pellet at several fixed temperatures. Inset: temperature dependence of the normalised current density defined as $J(T) / J\left(T_{0}\right)$ where $J(T)$ and $J\left(T_{0}\right)$ are the current densities values (measured at $90 \mu \mathrm{V}$ ) at $T$ and $T_{0}=2.9 \mathrm{~K}$, respectively. The red line is the least squared fit of the experimental points by Equation (1) (see the text) resulting in a $T_{C} \simeq 73 \mathrm{~K}$.

The nature of the detected fall of $\rho(T)$ was investigated by carrying out I-V characteristics at several fixed temperature. At $T \leq 30 \mathrm{~K}, \mathrm{I}-\mathrm{V}$ characteristics exhibits a linear behaviour even if the voltage drop remains relatively low (in the range of tens of microvolts) up to about $80 \mathrm{~mA}$, close to the maximum intensity achievable by the current source used in our system of measure. We found that the temperature dependence of the current density value at $90 \mu \mathrm{V}$, exhibits a smooth continuous decrease, raising $T$. We have carried out a least squared fit by a Ginzburg-Landau-like function, considering as dependent variable the normalised current density, $J_{n o r m}$, here defined as the ratio between the current density at $T, J(T)$, and the current density at the lowest temperature (i.e., $\left.T_{0}=2.9 \mathrm{~K}\right), J\left(T_{0}\right)$ :

$$
J_{\text {norm }}(T)=\left[1-\left(T / T_{C}\right)^{2}\right]^{3 / 2}\left[1+\left(T / T_{C}\right)^{2}\right]^{1 / 2}
$$

Application of Equation (1) has allowed estimating a superconducting critical temperature of $T_{C} \simeq$ $73 \mathrm{~K}$. This interesting result and the good best fitting by Equation (1) of the experimental points $J_{\text {norm }}(T)$ (Figure 6, inset) could suggest a proportionality between measured current density and the superconducting critical current density of the KPT sample.

Our finding of a bell shaped curve of the KPT resistivity suggests two different temperature dependent conduction mechanisms. Below RT, down to $\approx 150 \mathrm{~K}, \rho(T)$ presents a dielectric-like character, with an activation energy progressively decreasing from $1.29 \mathrm{eV}$, around room temperature, to about $2 \mathrm{meV}$, at $\simeq 150 \mathrm{~K}$ (Figure 5, inset). This behaviour could be explained assuming a trapping mechanism of charge carriers, as reported for polycrystalline thin films of $p$-terphenyl [50]. Moreover, the low value of $\rho(T)$ at RT could suggest a partial contribution of $K^{+}$ions to the electrical conduction that, anyway, is expected to rapidly decrease lowering $T$. Below $\approx 130 \mathrm{~K}, \rho(T)$ becomes metallic-like indicating an enhanced contribution to the conduction by electrons. The initial slow 
decrease of $\rho(T)$ is followed by a steeper fall at $\approx 100 \mathrm{~K}$ that, in addition to the residual value of $10^{-2}-10^{-3} \Omega \mathrm{cm}$ below $30 \mathrm{~K}$ (Figure 5), looks like that of a superconducting transition, that depending on the criterium taken into account, a superconducting transition temperature of $T_{C} \simeq 94 \mathrm{~K}$ [37] or $T_{C} \simeq 87 \mathrm{~K}$ (i.e., the $T$ corresponding to the $50 \%$ of the flat region of the $\rho(T)$ curve, here equals to $79 \Omega \mathrm{cm}$ ) can be defined, with a transition width of $\Delta T_{C} \simeq 22 \mathrm{~K}$, in both cases. The region between $150 \mathrm{~K}$ and $130 \mathrm{~K}$ marks the transition between these two conduction regimes.

The non-monotonic behaviour of the KPT resistivity shares important similarities with that observed in $\mathrm{K}_{x}$-picene, an organic molecule formed by 5 benzene rings fused together along their edges and doped by K-ions $(x \simeq 3$ ) [54]. Picene and $p$-terphenyl molecules, can be considered members of the same family of hydrocarbon superconductors.

Several findings measured in $\mathrm{K}_{x}$-picene pellets deserves to be mentioned and compared to those of KPT: a similar behaviour of the $\rho(T)$ curve, with an initial increase of $\rho(T)$ followed by a rapid decrease, lowering $T$ from RT; the highest resistivity value at the curve peak is $100 \Omega \mathrm{cm}(\simeq 80 \Omega \mathrm{cm}$ in $\mathrm{KPT}) ; \rho_{R T} \simeq 0.4 \Omega \mathrm{cm}$ (a few $\Omega \mathrm{cm}$ in $\left.\mathrm{KPT}\right) ;$ a superconducting transition width several kelvin wide $(\simeq 22 \mathrm{~K}$ in $\mathrm{KPT})$.

The transition width, in particular, and the huge drop of the KPT resistivity, are assigned to the granular nature of the KPT disks, fabricated by a compressed powder as for the $\mathrm{K}_{x}$-picene pellets [54]. Anyway, possible evidence of superconductivity in KPT compounds was inferred by magnetic susceptibility only [21,23], while ARPES and STM have experimentally proved the existence of a large superconducting energy gap [25,26], while previous resistivity measurements gave negative results [24]. On the other hand, magnetic susceptibility measurements indicating a superconducting state were reported for all hydrocarbon superconductors but $\mathrm{K}_{x}$-picene [54,55]. The resistivity drop by several orders of magnitude here observed in KPT represents a first possible direct evidence for superconductivity in this compound.

To successfully measure electrical properties of KPT, a percolating path connecting the electrical contacts is necessary. Hence, a large number of grains in the superconducting state will be involved to detect a drop of the resistivity. On the contrary, in the magnetic susceptibility, the superconducting features can be produced by not interconnected regions of the material giving more easily evidence of supercondutivity $[21,23]$. Our results support the hypothesis of a superconductivity state confined in isolated regions of the KPT sample that have probably prevented till now to observe any superconducting transition by electrical measurements. This is also due to the quite small values of the coherence length of the order of tens of nanometers that, due to the Josephson tunneling of Cooper pairs, would weakly couple KPT grains with a size of the order of microns. The possibility to observe a superconducting transition in the whole KPT disk, by electronic transport measurements, will depend on several factors such as purity of the pristine material, dimension of KPT crystallites, their density and distribution in the dielectric matrix. Achieving high- $T_{C}$ superconductivity will depend mainly on the doping level of the $p$-terphenyl molecule. Our Raman analysis has confirmed both the high quality and pureness of the undoped $p$-terphenyl and a successfull doping of KPT as well, that the AAS technique has established, in a quantitative way, to be of $\simeq 2 \mathrm{~K}$-ions for each $p$-terphenyl molecule. Additionally, the pressure applied to a KPT disk is expected to affect its electrical properties, due to an improved contact between KPT grains.

To explain the electrical behaviour detected below $\approx 130 \mathrm{~K}$, we hypothesise that KPT disks could be made of superconducting crystallites, with a distribution in their taille and $T_{C}$, dispersed in a dielectric matrix, as suggested by the presence of low-intensity Raman peaks due to residual grains of the undoped phase (see Figure 4, $T=203 \mathrm{~K})$. At low $T(<130 \mathrm{~K})$, under sufficient mechanical pressure, superconducting grains could have formed a percolative path between the metal contacts. Due to the $T_{C}$ distribution, the system will not undergo a narrow superconducting transition but will progressively reduce the resistivity, upon superconducting transition of single KPT crystallites. It is reasonable to suppose that at the lowest temperature not all crystallites have become superconducting or local inhomogeneity (see the spectrum at $203 \mathrm{~K}$ in Figure 4) has prevented a drop to zero of $\rho(T)$ [54] even 
if the lowest attained $\rho(T)$ values for our KPT material are $10^{-2}-10^{-3} \Omega \mathrm{cm}$. This last hypothesis is further supported by the lacking of a sudden transition to the normal state in the I-V curves, here substituted by a linear change of $I$ even at the lowest $T$ (see Figure 6). Both the shape of the transition and the lacking of a negligible $\rho(T)$ at $T_{C}$ can be considered a manifestation of the granular nature of the KPT material [54]. It is worthwhile noting that $T_{C}$ derived from I-V curves, fitting $I_{\text {norm }}$ by Equation (1), results comparable to that derived from resistivity measurements.

Percolative superconductivity is typical in granular superconducting materials [56]. Please note that intense Raman peaks, detected in the high frequency region of the spectrum of the KPT powder, even at $T=108 \mathrm{~K}$, justify a possible key role played by high energy phonons in the superconducting pairing. Even though the mechanism driving Cooper pairing in KPT systems is still unknown, the electron-phonon coupling with high energy phonons should play a key role in the high temperature superconductivity, as discussed above. In this framework, the density of electronic states (DOS) close to the Fermi energy is expected to influence the value of the critical temperature. As shown in Figure 2 of Ref. [57] the DOS of $p$-terphenyl has several peaks associated with van Hove singularities, because of multiple bands characterizing the anisotropic electronic structure. Electron doping by $\mathrm{K}$ atoms, increasing the Fermi energy toward positive values (right part of the DOS in Figure 2 of Ref. [57]), will determine a crossing of the van Hove singularities and an increase of the superconducting critical temperature. Our doping level of K-atoms, intermediate between the one in Ref. [15] $\left(T_{c}=7.2 \mathrm{~K}\right)$ and the other in Ref. [21] $\left(T_{\mathcal{C}}=123 \mathrm{~K}\right)$ is compatible with the intermediate value of $T_{C}$ around $91 \mathrm{~K}$ found in this work.

\section{Conclusions}

To conclude, the huge suppression of the resistivity of K-doped $p$-terphenyl measured in our experiments below $T=90 \mathrm{~K}$, of about four orders of magnitude in a temperature range of $\simeq 20 \mathrm{~K}$, represents a possible signature of a superconducting transition in this metallo-organic compound, providing additional support to the evidence of high- $T_{C}$ superconductivity found by magnetic susceptibility, ARPES and STM measurements. Future experiments on the electrical transport properties of KPT materials will focus on the magnetic field dependence of the resistivity curve $\rho(T, H)$, to study the expected suppression of the critical temperature when the external magnetic field is increased toward its upper critical value. Still we have to underline that this is a complex quantum material and samples are characterized by inhomogeneous concentrations of dopants and by a mixture of different coexisting phases.

Author Contributions: Conceptualization, N.P. and A.P.; methodology, N.P., A.T., M.M. and C.D.N.; validation, F.M., C.P. and A.B.; investigation, N.P., A.T., M.M. and C.D.N; writing-original draft preparation, N.P., A.T., M.M. and A.P. ; writing—review and editing, N.P., A.T., A.D.C., A.M., A.B., F.M., C.P. and A.P.; supervision, A.B. All authors have read and agreed to the published version of the manuscript.

Funding: This research received no external funding.

Acknowledgments: We thank David Neilson, Javid Rezvani and Meenakshi Sharma for useful discussions. We acknowledge the University of Camerino for providing technical and financial support. The School of Science and Technology and the Physics Division are acknowledged for their contribution to the installation of the microRaman and the Helium closed cycle cryostat equipments .

Conflicts of Interest: The authors declare that they have no conflict of interest.

\section{References}

1. Romero, F.D.; Pitcher, M.J.; Hiley, C.I.; Whitehead, G.F.S.; Kar, S.; Ganin, A.Y.; Antypov, D.; Collins, C.; Dyer, M.S.; Klupp, G.; Colman, R.H.; Prassides, K.; Rosseinsky, M.J. Redox-controlled potassium intercalation into two polyaromatic hydrocarbon solids. Nat. Chem. 2017, 9, 644. [CrossRef] [PubMed]

2. Mitsuhashi, R.; Suzuki, Y.; Yamanari, Y.; Mitamura, H.; Kambe, T.; Ikeda, N.; Okamoto, H.; Fujiwara, A.; Yamaji, M.; Kawasaki, N.; Maniwa, Y.; Kubozono, Y. Superconductivity in alkali-metal-doped picene. Nature 2010, 464, 76-79. [CrossRef] 
3. Rietveld, H.M.; Maslen, E.N.; Clews, C.J.B. An x-ray and neutron diffraction refinement of the structure of p-terphenyl. Acta Crystal. B Struct. Crystallog. Cryst. Chem. 1970, 26, 693. [CrossRef]

4. Baudour, J.L.; Cailleau, H.; Yelon, W.B. Structural phase transition in polyphenyls. IV. double-well potential in the disordered phase of $p$-terphenyl from neutron $(200 \mathrm{~K})$ and x-ray (room-temperature) diffraction data. Acta Crystal. B Struct. Crystallog. Cryst. Chem. 1977, 33, 1773. [CrossRef]

5. Cailleau, H.; Heidemann, A.; Zeyen, C.M.E. Observation of critical slowing down at the structural phase transition in $p$-terphenyl by high-resolution neutron spectroscopy. J. Phys. C Solid State Phys. 1979, 12 , L411. [CrossRef]

6. Lechner, R.E.; Toudic, B.; Cailleau, H. Observation of the effects of critical phenomena in paraterphenyl on quasielastic incoherent neutron spectra. J. Phys. C Sol. State Phys. 1984, 17, 405. [CrossRef]

7. Cailleau, H.; Baudour, J.L.; Meinnel, J.; Dworkin, A.; Moussa, F.; Zeyen, C.M.E. Double-well potentials and structural phase-transitions in polyphenyls. Faraday Discus. Chem. Soc. 1980, 69, 7. [CrossRef]

8. Baranyai, A.; Welberry, T.R. Molecular dynamics simulation study of solid polyphenyls: Structures determined by the interplay between intra- and intermolecular forces. Mol. Phys. 1992, 75, 867. [CrossRef]

9. Goossens, D.J.; Beasley, A.G.; Welberry, T.R.; Gutmann, M.J.; Piltz, R.O. Neutron diffuse scattering in deuterated para-Terphenyl, C 18 D 14. J. Phys. Cond. Mat. 2009, 21, 124204. [CrossRef]

10. Rice, A.P.; Tham, F.S.; Chronister, E.L. A temperature dependent x-ray study of the Order-Disorder enantiotropic phase transition of p-terphenyl. J. Chem. Crystal. 2013, 43, 14. [CrossRef]

11. Pinto, N.; Rezvani, S.J.; Favre, L.; Berbezier, I.; Fretto, M.; Boarino, L. Geometrically induced electron-electron interaction in semiconductor nanowires. Appl. Phys. Lett. 2016. [CrossRef]

12. Rezvani, S.J.; Gunnella, R.; Neilson, D.; Boarino, L.; Croin, L.; Aprile, G.; Fretto, M.; Rizzi, P.; Antonioli, D.; Pinto, N. Effect of carrier tunneling on the structure of Si nanowires fabricated by metal assisted etching. Nanotechnology 2016. [CrossRef] [PubMed]

13. Mazziotti, M.V.; Valletta, A.; Campi, G.; Innocenti, D.; Perali, A.; Bianconi, A. Possible Fano resonance for high-Tc multi-gap superconductivity in p-Terphenyl doped by $\mathrm{K}$ at the Lifshitz transition. EPL (Europhys. Lett.) 2017, 118, 37003. [CrossRef]

14. Barba, L.; Chita, G.; Campi, G.; Suber, L.; Bauer, E.M.; Marcelli, A.; Bianconi, A. Anisotropic thermal expansion of $p$-terphenyl: A self-assembled supramolecular array of poly-p-phenyl nanoribbons. J. Supercond. Novel Magnet. 2018, 31, 703. [CrossRef]

15. Wang, R.S.; Gao, Y.; Huang, Z.B.; Chen, X.J. Superconductivity in p-terphenyl. arXiv 2017, arXiv:1703.05803.

16. Huang, G.; Zhong, G.H.; Wang, R.S.; Han, J.X.; Lin, H.Q.; Chen, X.J. Superconductivity and phase stability of potassium-doped p-quinquephenyl. Carbon 2019, 143, 837. [CrossRef]

17. Yan, J.F.; Zhong, G.H.; Wang, R.S.; Zhang, K.; Lin, H.Q.; Chen, X.J. Superconductivity and Phase Stability of Potassium-Intercalated p-Quaterphenyl. J. Phys. Chem. Lett. 2019, 10, 40. [CrossRef]

18. Zhong, G.H.; Yang, D.Y.; Zhang, K.; Wang, R.S.; Zhang, C.; Lin, H.Q.; Chen, X.J. Superconductivity and phase stability of potassium-doped biphenyl. Phys. Chem. Chem. Phys. 2018, 20, 25217. [CrossRef]

19. Gao, Y.; Wang, R.S.; Wu, X.L.; Cheng, J.; Deng, T.G.; Yan, X.-W.; Huang, Z.B. Searching superconductivity in potassium-doped $p$-terphenyl. Acta Phys. Sin. 2016, 65, 077402.

20. Wang, R.S.; Gao, Y.; Huang, Z.B.; Chen, X.J. Superconductivity at $43 \mathrm{~K}$ in a single C-C bond linked terphenyl. arXiv 2017, arXiv:1703.05804.

21. Wang, R.S.; Gao, Y.; Huang, Z.B.; Chen, X.J. Superconductivity above 120 kelvin in a chain link molecule. arXiv 2017, arXiv:1703.06641.

22. Liu, W.; Lin, H.; Kang, R.; Zhang, Y.; Zhu, X.; Wen, H.H. Magnetization of potassium doped $p$-terphenyl and p-quaterphenyl by high pressure synthesis. Phys. Rev. B 2017, 96, 224501. [CrossRef]

23. Neha, P.; Bhardwaj, A.; Sahu, V.; Patnaik, S. Facile synthesis of potassium intercalated $p$-terphenyl and signatures of a possible high $\mathrm{T}_{c}$ phase. Phys. C Supercond. App. 2018, 554, 1. [CrossRef]

24. Carrera, M.; McDonald, J.L.; Untiedt, C.; García-Hernández, M.; Mompean, F.; Vergés, J.A.; Guijarro, A. Characterization of Main Phase in $\mathrm{K}_{x} \mathrm{p}$-Terphenyl and Its Largest Congener $\mathrm{K}_{x}$ poly(p-phenylene): A Report of Their Magnetic and Electric Properties. J. Phys. Chem. C 2019, 123, 5264-5272. [CrossRef]

25. Li, H.; Zhou, X.; Parham, S.; Nummy, T.; Griffith, J.; Gordon, K.; Chronister, E.L.; Dessau, D.S. Spectroscopic evidence of low-ernergy gaps persisting up to 120 Kelvin in surface-doped p-terphenyl cristals. Phys. Rev. B 2019, 100, 064511. [CrossRef] 
26. Ren, M.Q.; Chen, W.; Liu, Q.; Chen, C.; Qiao, Y.J.; Chen, Y.J.; Zhou, G.; Zhang, T.; Yan, Y.J.; Feng, D.L. Observation of novel gapped phases in potassium doped single layer $p$-terphenyl on Au (111). Phys. Rev. B 2019, 99, 045417. [CrossRef]

27. Perali, A.; Pieri, P.; Strinati, G.C.; Castellani, C. Pseudogap and spectral function from superconducting fluctuations to the bosonic limit. Phys. Rev. B 2002, 66, 024510. [CrossRef]

28. Palestini, F.; Perali, A.; Pieri, P.; Strinati, G.C. Dispersions, weights, and widths of the single-particle spectral function in the normal phase of a Fermi gas. Phys. Rev. B 2012, 85, 024517. [CrossRef]

29. Gaebler, J.P.; Stewart, J.T.; Drake, T.E.; Jin, D.S.; Perali, A.; Pieri, P.; Strinati, G.C. Observation of pseudogap behaviour in a strongly interacting Fermi gas. Nat. Phys. 2010, 6, 569. [CrossRef]

30. Marsiglio, F.; Pieri, P.; Perali, A.; Palestini, F.; Strinati, G.C. Pairing effects in the normal phase of a two-dimensional Fermi gas. Phys. Rev. B 2015, 91, 054509. [CrossRef]

31. Salasnich, L.; Shanenko, A.A.; Vagov, A.; Aguiar, J.A.; Perali, A. Screening of pair fluctuations in superconductors with coupled shallow and deep bands: A route to higher-temperature superconductivity. Phys. Rev. B 2019, 100, 064510. [CrossRef]

32. Tajima, H.; Yerin, Y.; Perali, A.; Pieri, P. Enhanced critical temperature, pairing fluctuation effects, and BCS-BEC crossover in a two-band Fermi gas. Phys. Rev. B 2019, 99, 180503(R). [CrossRef]

33. Valletta, A.; Bianconi, A.; Perali, A.; Saini, N.L. Electronic and superconducting properties of a superlattice of quantum stripes at the atomic limit. Z. Phys. B 1997, 104, 707. [CrossRef]

34. Fabrizio, M.; Qin, T.; Naghavi, S.S.; Tosatti, E. Two-Band s \pm Strongly Correlated Superconductivity in K3 p-Terphenyl ? arXiv 2017, arXiv:1705.05066.

35. Connelly, N.G.; Geiger, W.E. Chemical redox agent for organometallic chemistry. Chem. Rev. 1996, 96, 877. [CrossRef] [PubMed]

36. Zhong, G.H.; Wang, X.H.; Wang, R.S.; Han, J.X.; Zhang, C.; Chen, X.J.; Lin, H.Q. Structural and Bonding Characteristics of Potassium-Doped p-Terphenyl Superconductors. J. Phys. Chem. C 2018, 122, 3801. [CrossRef]

37. Pinto, N.; Rezvani, S.J.; Perali, A.; Flammia, L.; Milosevic, M.V.; Fretto, M.; Cassiago, C.; Leo, N.D. Dimensional crossover and incipient quantum size effects in superconducting niobium nanofilms. Sci. Rep. 2018, 8, 4710. [CrossRef]

38. Rezvani, S.J.; Perali, A.; Fretto, M.; Leo, N.D.; Flammia, L.; Milosevic, M.V.; Nannarone, S.; Pinto, N. Substrate-Induced Proximity Effect in Superconducting Niobium Nanofilms. Cond. Matter 2019, 4, 4. [CrossRef]

39. Bolton, B.A.; Prasad, P.N. Phase transitions in polyphenyls: Raman spectra of $p$-terphenyl and p-quaterphenyl in the solid state. Chem. Phys. 1978, 35, 331. [CrossRef]

40. da Costa, A.A.; Amado, A.; Becucci, M.; Kryschi, C. Order-disorder phase transition in $p$-terphenyl and p-terphenyl: Tetracene doped crystals as studied by Raman spectroscopy. J. Mol. Struct. 1997, 416, 69. [CrossRef]

41. Zhang, K.; Wang, R.; Chen, X. Vibrational Properties of p-terphenyl. J. Phys. Chem. A 2018, 122, 6903. [CrossRef] [PubMed]

42. Baudour, J.L.; Delugeard, Y.; Cailleau, H. Transition structurale dans les polyphényles. I. Structure cristalline de la phase basse température du p-terphényle à 113 K. Acta Crystallogr. Sect. B 1976, 32, 150-154. [CrossRef]

43. Brédas, J.; Thémans, B.; Fripiat, J.; André, J.; Chance, J.R. An ab-initio study of the geometry and electronic-structure modifications upon doping. Phys. Rev. B 1984, 29, 6761. [CrossRef]

44. Furukawa, Y. Electronic Absorption and Vibrational Spectroscopies of Conjugated Conducting Polymers. J. Phys. Chem. 1996, 100, 15644. [CrossRef]

45. Péres, L.; Spiesser, M.; Froyer, G. Reduction of $p$-terphenyl, p-quaterphenyl and p-sexiphenyl using alkali metal in liquid ammonia: Process and characterization of the reduced compounds. Synth. Met. 2005, 155, 450. [CrossRef]

46. Furukawa, Y.; Ohtsuka, H.; Tasumi, M. Raman studies of polarons and bipolarons in sodium-doped poly-p-phenylene. Synth. Met. 1993, 55, 516. [CrossRef]

47. Dubois, M.; Froyer, G.; Louarn, G.; Billaud, D. Raman spectroelectrochemical study of sodium intercalation into poly(p-phenylene). Spectrochim. Acta A Mol. Biomol. Spectros. 2003, 59, 1849. [CrossRef]

48. Chakraverty, B.K. Bipolarons and superconductivity. J. Phys. 1981, 42, 1351.:019810042090135100. [CrossRef]

49. Alexandrov, A.; Ranninger, J. Bipolaronic superconductivity. Phys. Rev. B 1981, 24, 1164. [CrossRef] 
50. Lipinski, A.; Mycielski, W.; Swiatek, J. Charge carrier transport and d.c. conductivity in thin polycrystalline p-terphenyl films. J. Phys. Chem. Solids 1980, 41, 455. [CrossRef]

51. Staryga, E.; Swiatek, J. The electrical conductivity in thin polycrystalline $p$-terphenyl films. Thin Sol. Films 1979, 56, 311. [CrossRef]

52. Tkaczyk, S.W. Electrical conductivity of the polycrystalline films of p-terphenyl. Proc. SPIE 1999, $3725,232$. [CrossRef]

53. Bizzarri, P.C.; Casa, C.D.; Pietra, S. Electrical conductivity of o-, m-, and p-terphenyls. Z. Naturforsch 1973, 28b, 331. [CrossRef]

54. Teranishi, K.; He, X.; Sakai, Y.; Izumi, M.; Goto, H.; Eguchi, R.; Takabayashi, Y.; Kambe, T.; Kubozono, Y. Observation of zero resistivity in K-doped picene. Phys. Rev. B 2013, 87, 060505. [CrossRef]

55. Kubozono, Y.; Goto, H.; Jabuchi, T.; Yokoya, T.; Kambe, T.; Sakai, Y.; Izumi, M.; Zheng, L.; Hamao, S.; Nguyen, H.L.; Sakata, M.; Kagayama, T.; Shimizu, K. Superconductivity in aromatic hydrocarbons. Physica C 2015, 514, 199. [CrossRef]

56. Ponta, L.; Andreoli, V.; Carbone, A. Superconducting-insulator transition in disordered Josephson junctions networks. Eur. Phys. J. B 2013, 86, 24. [CrossRef]

57. Geilhufe, R.M.; Borysov, S.S.; Kalpakchi, D.; Balatsky, A.V. Towards novel organic high-Tc superconductors: Data mining using density of states similarity search. Phys. Rev. Mater. 2018, 2, 024802. [CrossRef]

Publisher's Note: MDPI stays neutral with regard to jurisdictional claims in published maps and institutional affiliations.

(C) 2020 by the authors. Licensee MDPI, Basel, Switzerland. This article is an open access article distributed under the terms and conditions of the Creative Commons Attribution (CC BY) license (http://creativecommons.org/licenses/by/4.0/). 